\title{
Evaluation of the Correlation Between tTG-IgA Titer and Duodenal Biopsy Findings in Children With Suspected Celiac Disease
}

\author{
Mitra-Azra Aldaghi, ${ }^{1}$ Seyed-Mohsen Dehghani, ${ }^{1, *}$ and Mahmood Haghighat ${ }^{1}$ \\ ${ }^{1}$ Department of Pediatrics, Shiraz University of Medical Sciences, Shiraz, IR Iran \\ *Corresponding author: Seyed-Mohsen Dehghani, Department of Pediatrics, Shiraz University of Medical Sciences, Shiraz, IR Iran. E-mail: dehghanism@sums.ac.ir \\ Received 2015 July 22; Revised 2015 October 21; Accepted 2015 November 7.
}

\begin{abstract}
Background: Celiac disease is an immune-mediated inflammation of the small intestine caused by sensitivity to dietary gluten in genetically sensitive individuals.

Objectives: In this study, we aimed to evaluate the predictive value of tissue transglutaminase (tTG) antibodies for the diagnosis of celiac disease in a pediatric population in order to determine if duodenal biopsy can be avoided.

Patients and Methods: The subjects were selected among individuals with probable celiac disease, referring to a gastrointestinal clinic. After physical examinations and performing tissue transglutaminase-immunoglobulin A(tTG-IgA) tests, upper endoscopy was performed if serological titer was higher than $18 \mathrm{IU} / \mathrm{mL}$. Therapy started according to pathologic results.

Results: The sample size was calculated to be 121 subjects ( 69 female and 52 male subjects); the average age of subjects was 8.4 years. A significant association was found between serological titer and pathologic results; in other words, subjects with high serological titer had more positive pathologic results for celiac disease, compared to others $(\mathrm{P}<0.001)$. Maximum sensitivity $(65 \%)$ and specificity $(65.4 \%)$ were achieved at a serological titer of $81.95 \mathrm{IU} / \mathrm{ml}$; the calculated accuracy was lower in comparison with other studies. As the results indicated, lower antibody titer was observed in patients with failure to gain weight and higher antibody titer was reported in diabetic patients.

Conclusions: As the results indicated, a single serological test (tTg-IgA test) was not sufficient for avoiding intestinal biopsy.
\end{abstract}

Keywords: Celiac Disease, Diagnostic Accuracy, tTG-IgA, Small Bowel Biopsy

\section{Background}

Celiac disease (CD) is an immune-mediated inflammation of the small intestine caused by sensitivity to dietary gluten in genetically sensitive individuals. The disorder is common, occurring in 0.5 to 1 percent of the general population in most countries (1). Overall, the global distribution of the disease seems to parallel the distribution of HLA genotypes that predispose to CD, provided that the population is also exposed to gluten (2). The Naspghan recommends serologic screening of $\mathrm{CD}$ be performed in the following groups of children with a gluten-containing diet (1). Patients with failure to thrive, persistent diarrhea, chronic constipation, recurrent abdominal pain, or vomiting, Dental enamel hypoplasia of permanent teeth, idiopathic short stature, significant pubertal delay, iron deficiency anemia not responsive to supplementation, dermatitis herpeticformis-like rash, recurrent aphthous stomatitis, fracture not explained by the level of trauma, abnormal liver biochemical tests, chronic fatigue, and all members of the following high-risk groups: first-degree relatives of patients with $\mathrm{CD}$, autoimmune thyroiditis, type 1 diabetes, Down syndrome, Turner syndrome, Williams syndrome, selective IgA deficiency, and autoimmune hepatitis (3).
Diagnosis of CD for most patients is recommended with measuring IgA antibodies to human recombinant tissue transglutaminase (tTG). This test is highly specific and sensitive. With using second-generation ELISA technology, the sensitivity and specificity of anti-tTG antibodies for biopsy-proven CD are generally above 96 percent (1). Measurement of IgA antibodies to endomysium are equally accurate, but this test is more expensive and somewhat dependent on operator interpretation (4). Approximately 2 percent of children with CD have previously unrecognized IgA deficiency. Therefore, total IgA should be measured in children with negative results of IgA-tTG but a high clinical suspicion of CD (5).

All individuals with positive tTG antibodies or anti endomysial antibodies should have an intestinal biopsy to establish the diagnosis of CD. Multiple biopsies should be taken from the distal duodenum and duodenal bulb and interpreted by an expert pathologist; the disease may have a patchy distribution (6).

The assessment of intestinal biopsies requires a certain level of expertise and skill, and the diagnostic accuracy can be affected by variability in sample quality and subjective interpretation (7). As the difficulties in histological diagno-

Copyright (c) 2016, Growth \& Development Research Center. This is an open-access article distributed under the terms of the Creative Commons Attribution-NonCommercial 4.0 International License (http://creativecommons.org/licenses/by-nc/4.0/) which permits copy and redistribute the material just in noncommercial usages, provided the original work is properly cited. 
sis of CD in clinical practice have been well documented (8), the appropriate use of simpler and more accurate tools would add reliability to the diagnosis of $\mathrm{CD}$. A new diagnostic standard based on serology alone, which could accurately detect patients in any clinical setting, has been previously suggested (9). Thus, based on the very high positive predict values (PPVs) of reliable serological tests, some authors have proposed that intestinal biopsy could no longer be mandatory for the diagnosis of $C D$ in some patients.

\section{Objectives}

Our aim in this prospective study was to explore the performance of serology-based algorithms that can potentially obviate the need for intestinal biopsies for diagnosis.

\section{Patients and Methods}

The subjects were selected among individuals with suspected CD (with symptoms such as chronic diarrhea (3 cases), chronic abdominal pain (3 cases), failure to gain weight (52 cases), and short stature (32 cases), referring from other clinics, and high-risk individuals, namely cases with type 1 diabetes ( 24 cases) and first-degree relatives of patients with CD (1 case). The subjects were evaluated after physical examinations.
In total, 121 subjects (69 female and 52 male subjects) within the age range of 2 to 18 years were included in the study. Patients under the age of 2 years were eliminated from the study, given the low accuracy of serologic screening with tTG-IgA at this age.

In patients, tTg-IgA and total IgA tests were performed in a single laboratory. The new generation of Aeskulisa kit (Germany), along with ELISA method, was applied for all patients. A titer of $18 \mathrm{IU} / \mathrm{mL}$ or higher was considered positive. Upper endoscopy, along with duodenal biopsy (biopsy from bulb and discrete sites of duodenum), was performed on 5 samples. The samples were interpreted by an expert pathologist and findings were classified based on Marsh-Oberhuber categorization. According to pathologic findings, therapy was started.

We used the area under the receiver operating characteristic (ROC) curve to investigate the hypothesis and SPSS (Ver.11) for the statistical analyses.

\section{Results}

The subjects included 121 individuals within the age range of 2 - 18 years. The number of male and female subjects was not significantly different $(\mathrm{P}=0.804)$.

Among 121 cases, 40 subjects (33.1\%) had positive pathologic results for $\mathrm{CD}$ and 81 (66.9\%) had negative pathologic results. The lowest tTG-IgA titer was $19 \mathrm{~mL} / \mathrm{IU}$ and the highest was $980 \mathrm{~mL} / \mathrm{IU}$ (Table 1).

\begin{tabular}{|c|c|c|}
\hline Variables & Suspected Group & High Risk Group \\
\hline \multicolumn{3}{|l|}{ Gender } \\
\hline Female & $56(58.33)$ & $13(52.00)$ \\
\hline Male & $40(41.67)$ & $12(48.00)$ \\
\hline Age $($ mean \pm SD $)$ & $8.22 \pm 3.82$ & $9.16 \pm 3.24$ \\
\hline \multicolumn{3}{|l|}{ Chronic diarrhea } \\
\hline No & $93(96.88)$ & $25(100.00)$ \\
\hline Yes & $3(3.13)$ & $0(0)$ \\
\hline \multicolumn{3}{|c|}{ Failure to gain weight } \\
\hline No & $44(45.83)$ & $25(100.00)$ \\
\hline yes & $52(54.17)$ & $0(0)$ \\
\hline \multicolumn{3}{|c|}{ Chronic abdominal pain } \\
\hline No & $93(96.88)$ & $25(100.00)$ \\
\hline Yes & $3(3.13)$ & $0(0)$ \\
\hline \multicolumn{3}{|l|}{ Short stature } \\
\hline No & $64(66.67)$ & $25(100.00)$ \\
\hline Yes & $32(33.33)$ & $0(0)$ \\
\hline \multicolumn{3}{|l|}{ Pathology result } \\
\hline Negative & $65(61.71)$ & $16(66.94)$ \\
\hline Positive & $31(32.29)$ & $9(33.06)$ \\
\hline Serology titer & $109.142 \pm 133.217$ & $183.524 \pm 185.182$ \\
\hline
\end{tabular}

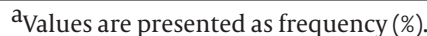


Among 121 patients, 25 cases were in the high-risk group, which included patients with diabetes mellitus (24 cases) and first-degree relatives of patients with CD (1 case). In the high-risk group, 9 patients had positive pathologic results, with mean serological level of $217 \mathrm{I} \mathrm{U} / \mathrm{mL}$ and standard deviation 129.6, while 16 subjects had negative pathologic results with mean serological level of $164.6 \mathrm{I}$ U/ $\mathrm{mL}$ and SD 211. Despite the lowert TG-IgA titer in subjects with negative pathologic results, compared to those with positive pathology, the difference was not statistically significant $(\mathrm{P}=0.121)$.

The serological titer was higher in the high-risk group (mean $=183$ and SD 185.1), compared to other subjects (mean = 109 and SD 133.2); also, the difference between the high-risk group and other patients was statistically significant $(\mathrm{P}=0.007)$. In the high-risk group, by using receiver operating characteristic (ROC) curve, the cut-off point for serological titer was $126 \mathrm{IU} / \mathrm{mL}$ (sensitivity $7 \%$ and specificity $75 \%$ ).

According to ESPGHAN guidelines (5), a serological titer of $\geq 3$ upper limit normal (ULN) is considered for avoiding biopsy in the high-risk group. So with serology titer of 54 ( $\geq 3$ ULN, normal: 18 ), sensitivity $88.9 \%$ and specificity $9 \%$ was concluded. The area under ROC curve was 0.694 and $\mathrm{p}$ value was estimated at 0.113 .

A statistically significant relation was observed between serological titer and positive pathologic results in patients with suspected CD. In other words, patients with positive pathologic results had higher serological titers (mean = $190 \mathrm{IU} / \mathrm{mL}$ and SD 180.5) and $\mathrm{P}<0.001$.

According to ROC curve, the area under the curve was $0.073(\mathrm{P}<0.001)$.

Maximum sensitivity and specificity were estimated at $65 \%$ and $65.4 \%$ with a serological titer of $81.9 \mathrm{IU} / \mathrm{mL}$, respectively (serologic titers above and lower this titer (81.9 IU/mL) were not suitable for maximum sensitivity and specificity). Among patients with positive pathologic results (40 individuals), 26 (65\%) cases had a serological titer of $\geq 81.95 \mathrm{IU} / \mathrm{mL}$; if the titer was higher or equal to $81.95 \mathrm{IU} / \mathrm{mL}$, pathologic results were considered positive.

Intestinal biopsy could be avoided in $21.48 \%$ of cases witha positive predictive value (PPV) of $48.15 \%$ and a negative predictive value (NPV) of 79.10\%.

According to ESPGHAN guidelines (5), a serological titer equal to or higher than 10 ULN eliminates the need for biopsy in individuals suspected for CD. Therefore, based on a titer of 180, intestinal biopsy could be avoided in $14.4 \%$ of cases with PPV $=70.8$ and NPV $=76.29$; titer sensitivity was estimated at $42 \%$ and specificity was found to be $14 \%$.

On the other hand, a significant correlation was observed between failure to gain weight and serological titer in patients. In fact, subjects with failure to gain weight had lower serological titer (mean serological titer of 93.4 with SD 95), compared to subjects without problems in gaining weight (mean serological titer of 147.9 with SD 174.9) $(\mathrm{P}=0.045)$.

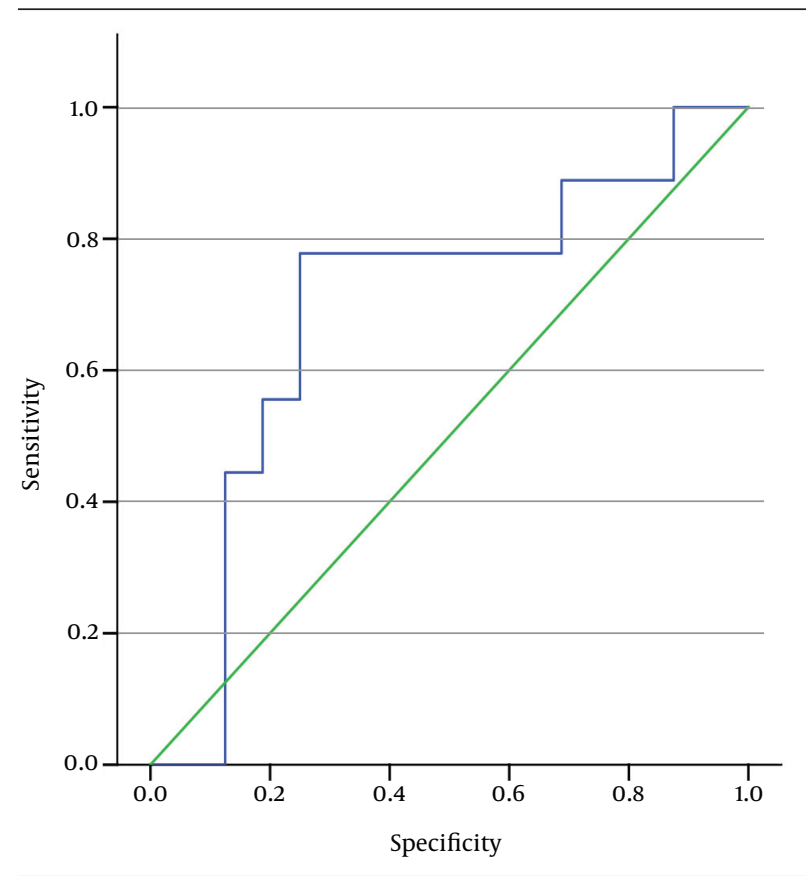

Figure 1. Statistical Analysis in Patients With Symptoms Suggestive of Celiac Disease in ROC Curve.

Figure 2. Comparison Between Serological Titer and Positive Pathologic Findings in ROC Curve

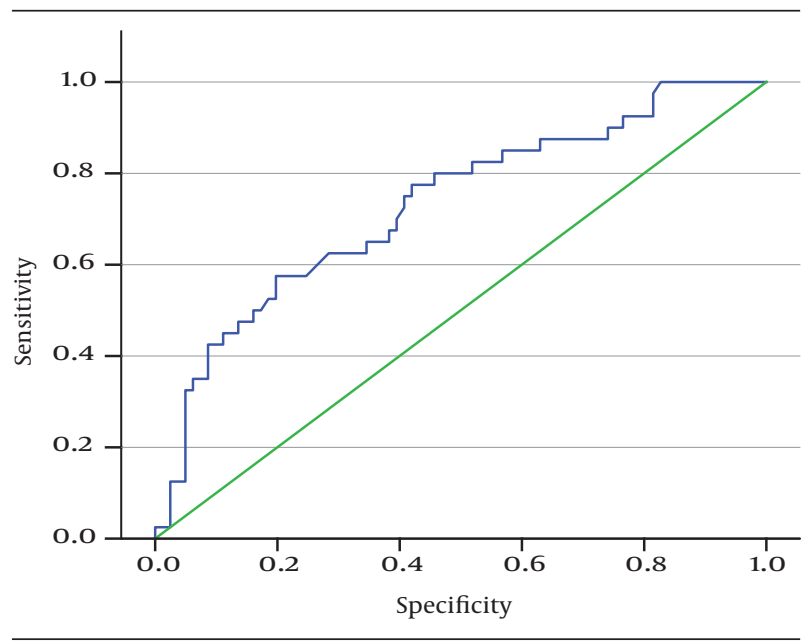

Diagonal segments are produced by ties.

Serological titer was not significantly correlated with chronic diarrhea or chronic abdominal pain in patients. Moreover, no significant relationship was observed between short stature of patients and serological titer.

However, serological titer in patients with diabetes mellitus (mean $=182.83$ with SD 189) was higher than that reported in other subjects (mean $=110$ with SD 132.8); this difference was statistically significant $(\mathrm{P}=0.012)$.

Evaluation of serological titer in first-degree relatives of celiac patients did not indicate any significance, given the low number of these subjects. 
No meaningful association was found between serological titer and type of pathology according to Kruskal-Wallis test; however, higher serological titer was correlated with higher pathologic grading.

The mean serological titer in patients with Marsh grade 2 (1 case) was $60.5 \mathrm{IU} / \mathrm{mL}$ and 174 - $182 \mathrm{IU} / \mathrm{mL}$ with SD 147 109.8 in patients with Marsh grade 3 (39 cases) $(\mathrm{P}=0.722$ ).

Moreover, serological titer and pathology grading were not significantly associated in patients with short stature, type 1 diabetes, or failure to gainweight.

Additionally, no statistically significant relation was observed between positive pathological results and short stature, chronic diarrhea, failure to gain weight, chronic abdominal pain, or type 1diabetes and first-degree relatives of patients with CD.

\section{Discussion}

Final diagnosis of CD is established via small bowel biopsy. In fact, this procedure is still considered the gold standard, despite the fact that morphological features are non-specific and other conditions can produce similar findings (10). However, according to conducted studies, diagnosis of CD can be made using serological titer to avoid intestinal biopsy; via this method, results can be obtained more rapidly and invasive operations can be avoided.

In previous studies, for the diagnosis of this condition, serological results were compared in cases with positive pathology in order to avoid biopsy in patients. In these studies, evaluation was performed using only one serological test (or several tests), combined with genetic studies. Intestinal biopsies could be avoided in $65 \%$ to $92 \%$ of cases in these studies (11).

In our study, one serological test (tTg-IgA), along with positive pathologic results, was used; therefore, in comparison with previous studies, fewer serological tests were performed (12). By selecting one serological test (tTgIgA), with higher (> 95\%) sensitivity and specificity were obtained and other tests such as endomysial antibodies (EMA) could be avoided. In fact, in comparison with EMA, the obtained results were equally accurate, though EMA test is more expensive and somewhat dependent on the operator's interpretation (13).

In our study, a statistically significant relation was observed between higher serological titers and positive pathologic results; however, in our study, higher serological titers had lower sensitivity and specificity, compared to other studies. With a serological titer of $81 \mathrm{IU} / \mathrm{mL}$, specificity and specificity were estimated at $65 \%$ and $65.4 \%$, respectively; this finding suggests that further studies are required.

In our study, application of a single serological test (tTgIgA) for the diagnosis of CD was controversial, contrary to other studies $(11,14)$.

A statistically significant relation was found between positive pathologic results and serological titers. In fact, patients with positive pathologic results had higher serological titer (mean $190 \mathrm{IU} / \mathrm{mL}$ and SD 180.5), compared to patients with negative pathologic results (mean $91 \mathrm{IU} / \mathrm{mL}$ and SD 116.4) (11).

The high-risk group (patients with diabetes mellitus and first-degree relatives of patients with CD) had higher serological titers, compared to others; moreover, a significant relation was found. This finding (higher serological titers) was in match with ESPGHAN guidelines, which indicate that in high-risk individuals with lower serological titers ( $\geq 3$ ULN) in comparison with other patients suspected for CD ( $\geq 10$ ULN), biopsy can be avoided (5).

Patients with failure to gain weight (mean $93 \mathrm{IU} / \mathrm{mL}$ ) had lower serological titers, compared to other subjects (mean $147 \mathrm{IU} / \mathrm{mL}$ ); this finding suggests that more interventions are required for the diagnosis of $C D$ in these individuals. A similar finding was reported in patients with chronic diarrhea; however, considering the low number of these patients, confirmation of the findings needs further analysis.

Also, considering the low number of patients with chronic abdominal pain and chronic diarrhea, and firstdegree relatives of patients with $\mathrm{CD}$, further analysis is required. Moreover, in the high-risk group, serological titer in subjects with positive pathologic findings was higher than in patients with negative pathology; however, the difference was not statistically significant.

\subsection{Conclusion}

In our study, considering the lower sensitivity and specificity of serological titers and lower success in avoiding intestinal biopsy, in contrast with other studies, the utility of one serological test for the accurate diagnosis of $\mathrm{CD}$ is not sufficient and further analysis is required. To obtain the best serological algorithm, future studies need to evaluate as many tests and test combinations as possible. Furthermore, a diversity of patient populations needs to be evaluated by taking different relevant aspects (e.g., disease prevalence and the magnitude of intestinal mucosal damage) into consideration.

\section{References}

1. Hill ID, Dirks MH, Liptak GS, Colletti RB, Fasano A, Guandalini S, et al. Guideline for the diagnosis and treatment of celiac disease in children: recommendations of the North American Society for Pediatric Gastroenterology, Hepatology and Nutrition.J Pediatr Gastroenterol Nutr. 2005;40(1):1-19. [PubMed:15625418]

2. Cataldo F, Montalto G. Celiac disease in the developing countries: a new and challenging public health problem. World J Gastroenterol. 2007;13(15):2153-9. [PubMed:17465493]

3. Caprai S, Vajro P, Ventura A, Sciveres M, Maggiore G, Sigenp Study Group for Autoimmune Liver Disorders in Celiac Disease. Autoimmune liver disease associated with celiac disease in childhood: a multicenter study. Clin Gastroenterol Hepatol. 2008;6(7):803-6. doi:10.1016/j.cgh.2007.12.002. [PubMed:18258488]

4. Pittschieler K, Ladinser B. Coeliac disease: screened by a new strategy. Acta Paediatr Suppl. 1996;412:42-5. [PubMed: 8783755]

5. Husby S, Koletzko S, Korponay-Szabo IR, Mearin ML, Phillips A, Shamir R, et al. European Society for Pediatric Gastroenterol- 


\section{Aldaghi M et al.}

ogy, Hepatology, and Nutrition guidelines for the diagnosis of coeliac disease. J Pediatr Gastroenterol Nutr. 2012;54(1):136-60. doi: 10.1097/MPG.0b013e31821a23do. [PubMed: 22197856]

6. Levinson-Castiel R, Hartman C, Morgenstern S, Avitzur Y, Hirsch A, Rosenbach Y, et al. The role of duodenal bulb biopsy in the diagnosis of celiac disease in children. J Clin Gastroenterol. 2011;45(1):26-9. doi: 10.1097/MCG.0b013e3181e04d26. [PubMed: 20628309]

7. Ladas SD, Tsamouri M, Kouvidou C, Raptis SA. Effect of forceps size and mode of orientation on endoscopic small bowel biopsy evaluation. Gastrointest Endosc. 1994;40(1):51-5. [PubMed: 8163135]

8. Pinto Sanchez MI, Smecuol E, Vazquez H, Mazure R, Maurino E, Bai JC. Very high rate of misdiagnosis of celiac disease in clinical practice. Acta Gastroenterol Latinoam. 2009;39(4):250-3. [PubMed:20178253]

9. Sugai E, Moreno ML, Hwang HJ, Cabanne A, Crivelli A, Nachman F, et al. Celiac disease serology in patients with different pretest probabilities: is biopsy avoidable? World J Gastroenterol. 2010;16(25):3144-52. [PubMed: 20593499]
10. Green PH, Rostami K, Marsh MN. Diagnosis of coeliac disease Best Pract Res Clin Gastroenterol. 2005;19(3):389-400. doi:10.1016/j. bpg.2005.02.006. [PubMed: 15925844]

11. Vivas S, Ruiz de Morales JG, Riestra S, Arias L, Fuentes D, Alvarez $\mathrm{N}$, et al. Duodenal biopsy may be avoided when high transglutaminase antibody titers are present. World J Gastroenterol. 2009;15(38):4775-80. [PubMed: 19824110]

12. Burgin-Wolff A, Mauro B, Faruk H. Intestinal biopsy is not always required to diagnose celiac disease: a retrospective analysis of combined antibody tests. BMC Gastroenterol. 2013;13:19. doi: 10.1186/1471-230X-13-19. [PubMed: 23343249]

13. Lewis NR, Scott BB. Systematic review: the use of serology to exclude or diagnose coeliac disease (a comparison of the endomysial and tissue transglutaminase antibody tests). Aliment Pharmacol Ther. 2006;24(1):47-54. doi:10.1111/j.1365-2036.2006.02967.x. [PubMed:16803602]

14. Freeman HJ. Strongly positive tissue transglutaminase antibody assays without celiac disease. Can J Gastroenterol. 2004;18(1):25-8. [PubMed:14760428] 